TRANSACTIONS OF THE

AMERICAN MATHEMATICAL SOCIETY

Volume 361, Number 7, July 2009, Pages 3817-3828

S 0002-9947(09)04708-4

Article electronically published on March 4, 2009

\title{
WEAKLY HOLOMORPHIC MODULAR FORMS OF HALF-INTEGRAL WEIGHT WITH NONVANISHING CONSTANT TERMS MODULO $\ell$
}

\author{
D. CHOI
}

\begin{abstract}
Let $\ell$ be a prime and $\lambda, j \geq 0$ be an integer. Suppose that $f(z)=$ $\sum_{n} a(n) q^{n}$ is a weakly holomorphic modular form of weight $\lambda+\frac{1}{2}$ and that $a(0) \not \equiv 0(\bmod \ell)$. We prove that if the coefficients of $f(z)$ are not "welldistributed" modulo $\ell^{j}$, then

$$
\lambda=0 \text { or } 1 \quad\left(\bmod \frac{\ell-1}{2}\right) .
$$

This implies that, under the additional restriction $a(0) \not \equiv 0(\bmod \ell)$, the following conjecture of Balog, Darmon and Ono is true: if the coefficients of a modular form of weight $\lambda+\frac{1}{2}$ are almost (but not all) divisible by $\ell$, then either $\lambda \equiv 0\left(\bmod \frac{\ell-1}{2}\right)$ or $\lambda \equiv 1\left(\bmod \frac{\ell-1}{2}\right)$. We also prove that if $\lambda \not \equiv 0$ and 1 $\left(\bmod \frac{\ell-1}{2}\right)$, then there does not exist an integer $\beta, 0 \leq \beta<\ell$, such that $a(\ell n+\beta) \equiv 0(\bmod \ell)$ for every nonnegative integer $n$. As an application, we study congruences for the values of the overpartition function.
\end{abstract}

\section{INTRODUCTION}

Much is known about the divisibility of the Fourier coefficients of modular forms of integral weight. When the weight is half-integral, the situation is not as clear. The distribution of the coefficients of modular forms of half-integral weight in congruence classes has been studied by, among others, Balog, Darmon, and Ono [5], Ono and Skinner [14, 15, Brunier [6], Bruinier and Ono 7], and Ahlgren and Boylan [1, 2]. In this paper, we study congruences for weakly holomorphic modular forms of half-integral weight such that the constant terms of their $q$-expansions are not zero modulo a prime $\ell$.

Suppose that $\lambda$ is an integer and $N$ is a positive integer with $4 \mid N$. We denote the space of modular forms (resp. cusp forms) of weight $\lambda+\frac{1}{2}$ on the congruence subgroup $\Gamma_{0}(N)$ with Dirichlet character $\chi$ by $M_{\lambda+\frac{1}{2}}\left(\Gamma_{0}(N), \chi\right)$ (resp. $\left.S_{\lambda+\frac{1}{2}}\left(\Gamma_{0}(N), \chi\right)\right)$. Let $\mathcal{M}_{\lambda+\frac{1}{2}}\left(\Gamma_{0}(N)\right)$ be the space of weakly holomorphic modular forms of weight $\lambda+\frac{1}{2}$ on $\Gamma_{0}(N)$. Note that if $\lambda<0$, then

$$
S_{\lambda+\frac{1}{2}}\left(\Gamma_{0}(N), \chi\right)=M_{\lambda+\frac{1}{2}}\left(\Gamma_{0}(N), \chi\right)=\{0\} .
$$

For convenience, we define the following property.

Received by the editors May 1, 2007 and, in revised form, August 15, 2007.

2000 Mathematics Subject Classification. Primary 11F11, 11F33.

(C)2009 American Mathematical Society Reverts to public domain 28 years from publication 
Definition. Let $q:=e^{2 \pi i z}$. A weakly holomorphic modular form $f(z):=\sum_{n} a(n) q^{n}$ $\in\left(\mathbb{Z}\left[q^{-1}\right]\right)[[q]] \cap \mathcal{M}_{\lambda+\frac{1}{2}}\left(\Gamma_{0}(N)\right)$ is well-distributed for a natural number $M$ if $f(z)$ satisfies the following: for every integer $r$

$$
\sharp\{1 \leq n \leq X \mid a(n) \equiv r \quad(\bmod M)\} \gg_{r, M}\left\{\begin{array}{lll}
\frac{\sqrt{X}}{\log X} & \text { if } r \not \equiv 0 & (\bmod M), \\
X & \text { if } r \equiv 0 & (\bmod M) .
\end{array}\right.
$$

Recently it was proved in [7] and [2] that

$$
f(z):=\sum_{n=1}^{\infty} a(n) q^{n} \in S_{\lambda+\frac{1}{2}}\left(\Gamma_{0}(N), \chi\right) \cap \mathbb{Z}[[q]]
$$

has a special form

$$
f(z) \equiv \sum_{i=1}^{t} \sum_{m=1}^{\infty} a\left(n_{i} m^{2}\right) q^{n_{i} m^{2}} \quad(\bmod \ell)
$$

if $f(z)$ is not well-distributed for an odd prime $\ell^{j}$. From these results, it is a natural question to ask what can be said about modular forms of half-integral weight whose coefficients are supported on finitely many square classes modulo $\ell$. For the question in positive characteristic, Balog, Darmon and Ono gave the following conjecture.

Conjecture A (Conjecture A in [5]). Suppose that $\lambda$ is a nonnegative integer, that $N$ is a positive integer $4 \mid N$, and $\ell$ is an odd prime. If $f(z) \in M_{\lambda+\frac{1}{2}}\left(\Gamma_{1}(N)\right) \cap \mathbb{Z}[[q]]$ is a modular form whose coefficients are almost all (but not all) divisible by $\ell$, then either $\lambda \equiv 0\left(\bmod \frac{\ell-1}{2}\right)$ or $\lambda \equiv 1\left(\bmod \frac{\ell-1}{2}\right)$.

In 2] Ahlgren and Boylan proved that if $f(z)$ is a modular form whose coefficients are supported on finitely many square classes modulo $\ell$, then, as predicted by Conjecture $\mathrm{A}$, the values of $\lambda$ are restricted.

Theorem (Theorem 2 in [2]). Suppose that we have the following hypotheses:

(i) $\lambda \geq 2$ is an integer, $N$ is a positive integer with $4 \mid N$, and $\chi$ is a real Dirichlet character modulo $N$.

(ii) $\ell \geq 5$ is a prime such that $\ell \nmid N$.

(iii) $f(z)$ is a cusp form of half-integral weight such that

$$
f(z)=\sum_{n=1}^{\infty} a(n) q^{n} \in S_{\lambda+\frac{1}{2}}\left(\Gamma_{0}(N), \chi\right) \cap \mathbb{Z}[[q]] .
$$

(iv) $f(z) \not \equiv 0(\bmod \ell)$, and there are finitely many square-free integers $n_{1}, n_{2}, \ldots$, $n_{t}$ such that

$$
f(z) \equiv \sum_{i=1}^{t} \sum_{m=1}^{\infty} a\left(n_{i} m^{2}\right) q^{n_{i} m^{2}} \quad(\bmod \ell) .
$$

Write $\lambda=\bar{\lambda}+i_{\lambda}(\ell-1)$ with $0 \leq \bar{\lambda} \leq \ell-2$. Then the following are true:

(1) If $\ell \nmid n_{i}$ for some $i$, then

$$
\bar{\lambda} \leq 2 i_{\lambda}+1 .
$$

(2) If $\ell \mid n_{i}$ for all $i$ and $\bar{\lambda} \leq \frac{\ell-3}{2}$, then

$$
\bar{\lambda} \leq 2 i_{\lambda}-\frac{\ell-1}{2} .
$$


(3) If $\ell \mid n_{i}$ for all $i$ and $\bar{\lambda} \geq \frac{\ell-1}{2}$, then

$$
\bar{\lambda} \leq 2 i_{\lambda}+\frac{\ell+3}{2} .
$$

In the following theorem, we prove that, under the additional restriction that $f(z)=\sum_{n} a(n) q^{n} \in M_{\lambda+\frac{1}{2}}\left(\Gamma_{0}(N)\right)$ and $a(0) \not \equiv 0(\bmod \ell)$, Conjecture A is true.

Theorem 1. Suppose that $f(z):=\sum_{n=0}^{\infty} a(n) q^{n} \in M_{\lambda+\frac{1}{2}}\left(\Gamma_{0}(N)\right)$ and that $a(0) \not \equiv$ $0(\bmod \ell)$. Then Conjecture $A$ is true.

Remark 1.1. (1) It was shown in $[3]$ that Conjecture A is not true in general. For example, if $T(z):=1+2 \sum_{n=1}^{\infty} q^{n^{2}}$ and $\theta:=\frac{1}{2 \pi i} \frac{d}{d z}$, then there exists a modular form $g(z)$ of weight $\lambda+\frac{1}{2}$ such that $g(z) \equiv \theta^{n} T(z)(\bmod \ell)$ and $\lambda=(\ell+1) n$.

(2) Under the additional restriction that

$$
f(z):=\sum_{n=1}^{\infty} a(n) q^{n} \in S_{\lambda+\frac{1}{2}}\left(\Gamma_{0}(N)\right) \cap \mathbb{Z}[[q]]
$$

and $\lambda \leq \ell-2$, the results of [2] and [8] establish that Conjecture $\mathrm{A}$ is true.

Note that if a cusp form of half-integral weight is not well-distributed for $\ell^{j}$, then its coefficients are supported on finitely many square classes modulo $\ell$. So, our following theorem is an analog of the result of Ahlgren and Boylan (Theorem 2 in [2]) for weakly holomorphic modular forms of half-integral weight such that the constant terms of their $q$-expansions are not zero modulo $\ell$.

Theorem 2. Suppose that $\lambda$ is an integer, that $N$ is a positive integer $4 \mid N$, and $\ell \geq 5$ is a prime. Suppose that

$$
f(z):=\sum_{n} a(n) q^{n} \in \mathcal{M}_{\lambda+\frac{1}{2}}\left(\Gamma_{0}(N)\right) \cap\left(\mathbb{Z}\left[q^{-1}\right]\right)[[q]] .
$$

Suppose further that $a(0) \not \equiv 0(\bmod \ell)$ and that $f(z)$ is not well-distributed for $\ell^{j}$, where $j$ is a positive integer.

(1) If $\ell \nmid N$, then $\lambda \equiv 0$ or $1\left(\bmod \frac{\ell-1}{2}\right)$.

(2) If $j=1$, then $\lambda \equiv 0$ or $1\left(\bmod \frac{\ell-1}{2}\right)$.

Let $p(n)$ be the ordinary partition function. Ramanujan found the following congruences for $p(n)$ :

$$
\begin{aligned}
& p(5 n+4) \equiv 0 \quad(\bmod 5), \\
& p(7 n+5) \equiv 0 \quad(\bmod 7), \\
& p(11 n+6) \equiv 0 \quad(\bmod 11) .
\end{aligned}
$$

Moreover, he claimed that if $0 \leq \beta<\ell$ is an integer for which

$$
p(\ell n+\beta) \equiv 0 \quad(\bmod \ell)
$$

for every nonnegative integer $n$, then $(\ell, \beta) \in\{(5,4),(7,5),(11,6)\}$. This conjecture was proved in [1] by Ahlgren and Boylan. Suppose that $\beta \in\{0,1, \ldots, \ell-1\}$ and

$$
f(z):=\sum_{n} a(n) q^{n} \in \mathcal{M}_{\lambda+\frac{1}{2}}\left(\Gamma_{0}(N)\right) \cap\left(\mathbb{Z}\left[q^{-1}\right]\right)[[q]] .
$$


We say that there is a congruence for $f(z)$ at $(\ell, \beta)$ if $a(\ell n+\beta) \equiv 0(\bmod \ell)$ for every nonnegative integer $n$. Let $\eta(z):=q^{\frac{1}{24}} \prod_{n=1}^{\infty}\left(1-q^{n}\right)$. Since

$$
\frac{1}{\eta(24 z)}=\sum_{n=0}^{\infty} p(n) q^{24 n-1}
$$

the claim of Ramanujan implies that if there is a congruence for the weakly holomorphic modular form $\frac{1}{\eta(24 z)}$ at $(\ell, \beta)$, then $(\ell, \beta) \in\{(5,0),(7,0),(11,0)\}$. In the following theorem, we prove that if $a(0) \not \equiv 0(\bmod \ell)$ and there is a congruence for $f(z)$ at $(\ell, \beta)$, then, as Ramanujan's congruences for the ordinary partition function, the prime $\ell$ is restricted.

Theorem 3. Suppose that $\lambda$ is an integer, that $N$ is a positive integer $4 \mid N$, and $\ell \geq 5$ is a prime. Suppose that

$$
f(z):=\sum_{n} a(n) q^{n} \in \mathcal{M}_{\lambda+\frac{1}{2}}\left(\Gamma_{0}(N)\right) \cap\left(\mathbb{Z}\left[q^{-1}\right]\right)[[q]]
$$

such that $a(0) \not \equiv 0(\bmod \ell)$. If for a sufficiently large integer $M$ there is a congruence for $f(z) \mid U_{\ell^{m}}$ at $(\ell, \beta), 0 \leq \beta<\ell$, then $\lambda \equiv 0$ or $1\left(\bmod \frac{\ell-1}{2}\right)$.

As an application of our main theorems, we study congruences for the values of the overpartition function.

Example 1.2. The overpartition of a natural number $n$ is a partition of $n$ in which the first occurrence of a number may be overlined (see [9] for more about overpartition). Let $\bar{P}(n)$ be the number of the overpartition of an integer $n$. It turns out that

$$
\sum_{n=0}^{\infty} \bar{P}(n) q^{n}=\frac{\eta(2 z)}{\eta(z)^{2}}
$$

and that $\frac{\eta(2 z)}{\eta(z)^{2}} \in \mathcal{M}_{-\frac{1}{2}}\left(\Gamma_{0}(16)\right)$ (see $[9]$ ). So, using Theorems 2 and 3 , we have the following congruence properties for $\bar{P}(n)$. Suppose that $\ell \geq 7$ is a prime.

(1) Then, for every integer $r$ and $j>0$,

$$
\sharp\left\{1 \leq n \leq X \mid \bar{P}(n) \equiv r \quad\left(\bmod \ell^{j}\right)\right\} \gg_{r, \ell}\left\{\begin{array}{lll}
\frac{\sqrt{X}}{\log X} & \text { if } r \neq \equiv 0 \quad\left(\bmod \ell^{j}\right), \\
X & \text { if } r \equiv 0 \quad\left(\bmod \ell^{j}\right) .
\end{array}\right.
$$

(2) Then for a sufficiently large integer $M$ there does not exist an integer $\beta$, $0 \leq \beta<\ell$, such that $\bar{P}\left(\ell^{m}(\ell n+\beta)\right) \equiv 0(\bmod \ell)$ for every nonnegative integer $n$.

The proofs of our theorems are obtained by studying filtrations of modular forms modulo $\ell$ and the combinatorial properties of Tate cycles. We remark that much of our argument is based on the work of Bruinier and Ono [7] and of Ahlgren and Boylan [1], [3], 4].

This paper is organized as follows. In Section 2 we recall some basic properties of the filtration of a modular form modulo $\ell$. In Section 3 we give the proof of Theorem 2 In Section 4 we prove Theorems 1 and 3 


\section{MOdular FORMS MOD $\ell$}

Throughout this section, we fix an integer $N \geq 1$ and a prime $\ell \nmid N$. We will recall the definitions and results that will be needed for our proof. The theory of modular forms modulo $\ell$ was developed by Serre [16] and Swinnerton-Dyer [17] for forms of level 1. We will recall the definitions and results that will be needed for the next section. For proofs when $N \geq 4$ and $\ell \nmid N$, see Gross [11.

Denote by $M_{k}$ the space of modular forms of weight $k$ on $\Gamma_{1}(N)$ with integer coefficients. If $f \in M_{k}$, let $\tilde{f} \in(\mathbb{Z} / \ell \mathbb{Z})[[q]]$ be the (coefficient-wise) reduction of $f \bmod \ell$. We write $\widehat{M_{k}}:=\left\{\tilde{f} \mid f \in M_{k}\right\}$ for the space of weight $k$ modular forms modulo $\ell$ with level $N$.

The filtration $\omega(\tilde{f})$ of a modular form $\tilde{f} \in \widetilde{M_{k}}$ is defined to be

$$
\omega(\tilde{f}):=\inf \left\{k^{\prime} \mid \tilde{f} \in \widetilde{M_{k^{\prime}}}\right\} .
$$

If $f \in M_{k}$, we sometimes write $\omega(f)$ instead of $\omega(\tilde{f})$ if no confusion can arise. Note that $\omega(\tilde{f})=-\infty$ if and only if $f \equiv 0(\bmod \ell)$. If $f \in M_{k}, g \in M_{k^{\prime}}$, and $f \equiv g \not \equiv 0$ $(\bmod \ell)$, then we must have $k \equiv k^{\prime}(\bmod \ell-1)$. From this it follows that $\omega(\tilde{f}) \equiv k$ $(\bmod \ell-1)$.

For a positive integer $m$, the operator $U_{m}$ acts on $q$-expansions by

$$
\sum_{n} a(n) q^{n} \mid U_{m}=\sum_{n} a(m n) q^{n} .
$$

Note that if $T_{\ell}$ denotes the $\ell$-th Hecke operator on $M_{k}$, then $f\left|T_{\ell} \equiv f\right| U_{\ell}(\bmod \ell)$, so $U_{\ell}$ acts on the spaces $\widetilde{M_{k}}$ and $\omega(f) \geq \omega\left(f \mid U_{\ell}\right)$. We define the theta operator by its effect on Fourier expansions:

$$
\theta\left(\sum_{n} a(n) q^{n}\right)=\sum_{n} n a(n) q^{n} .
$$

We record some basic properties of the filtration of a modular form modulo $\ell$.

Lemma 2.1 (11], [14, [17]). Let $f, g \in \widetilde{M_{k}}$. Write $k=\bar{k}+i_{k}(\ell-1)$ with $0 \leq \bar{k} \leq$ $\ell-2$.

(1) If $f$ is not a constant modulo $\ell$, then $\omega(f)>0$.

(2) $\omega\left(f^{\ell}\right)=\ell \cdot \omega(f)$.

(3) $\bar{k} \leq \omega(f) \leq k$.

(4) $\omega(f+g) \leq \max \{\omega(f), \omega(g)\}$.

(5) $\omega(\theta f) \equiv k+\ell+1(\bmod \ell-1)$.

(6) Suppose that $f$ is not a constant modulo $\ell$. Then $\omega(\theta f) \leq \omega(f)+\ell+1$ with equality if and only if $\omega(f) \not \equiv 0(\bmod \ell)$.

\section{Proof of Theorem 2}

Recall that $T(z)=1+2 \sum_{n=1}^{\infty} q^{n^{2}} \in M_{\frac{1}{2}}\left(\Gamma_{0}(4)\right)$. We begin with a lemma.

Lemma 3.1. Suppose that $\lambda$ is a nonnegative integer, that $N$ is a positive integer $4 \mid N$, and $\ell \geq 5$ is a prime such that $\ell \nmid N$. Suppose that

$$
f(z):=\sum_{n=0}^{\infty} a(n) q^{n} \in M_{\lambda+\frac{1}{2}}\left(\Gamma_{1}(N)\right) \cap \mathbb{Z}[[q]]
$$


such that $a(0) \not \equiv 0(\bmod \ell)$, and that $\omega\left(f(z) T(z)^{\ell}\right) \leq \ell+\frac{\ell+1}{2}$. If $\omega\left(\theta\left(f(z) T(z)^{\ell}\right)\right)=$ $\omega\left(\theta^{\frac{\ell+1}{2}}\left(f(z) T(z)^{\ell}\right)\right)$, then $\lambda \equiv 0$ or $1\left(\bmod \frac{\ell-1}{2}\right)$.

Proof of Lemma 3.1. Note that $\omega\left(f(z) T(z)^{\ell}\right) \equiv \lambda+\frac{\ell+1}{2}(\bmod \ell-1)$. This implies that

$$
\begin{array}{lll}
\lambda \equiv 1 & \left(\bmod \frac{\ell-1}{2}\right) & \text { if } \omega\left(f(z) T(z)^{\ell}\right)=\ell+\frac{\ell+1}{2}, \\
\lambda \equiv 0 & \left(\bmod \frac{\ell-1}{2}\right) & \text { if } \omega\left(f(z) T(z)^{\ell}\right)=\ell .
\end{array}
$$

So, we assume that $\omega\left(f(z) T(z)^{\ell}\right) \neq \ell+\frac{\ell+1}{2}$ and $\omega\left(f(z) T(z)^{\ell}\right) \neq \ell$. Note that

$$
\theta\left(f(z) T(z)^{\ell}\right) \equiv \theta^{\frac{\ell+1}{2}}\left(f(z) T(z)^{\ell}\right) \quad(\bmod \ell) .
$$

Then, using Lemma 2.1 (6), we have that there is an integer $1 \leq t \leq \frac{\ell-1}{2}$ such that $\theta^{t}\left(f(z) T(z)^{\ell}\right) \equiv 0(\bmod \ell)$. Thus, we have

$$
\frac{\ell+1}{2} \leq \omega\left(f(z) T(z)^{\ell}\right) \leq \ell-1 .
$$

Let $G(z):=f(z) T(z)^{\ell}$ and $\omega(\theta G(z)):=\ell+r, r \in \mathbb{Z}$. Then $\frac{\ell+3}{2} \leq r \leq \ell$ by Lemma 2.1 (6). Now we consider the value $\omega\left(\theta^{\frac{\ell+1}{2}} G(z)\right)$. Using Lemma 2.1 (3) and (5), we obtain

$$
\omega\left(\theta^{\ell-r+2} G(z)\right)=\omega(\theta G(z))+(\ell-r+1)(\ell+1)-\alpha(\ell-1),
$$

where $\alpha$ is a nonnegative integer. Thus, Lemma 2.1 (6) implies that

$$
\omega\left(\theta^{\ell-r+2} G(z)\right)+\left(r-\frac{\ell+3}{2}\right)(\ell+1) \geq \omega\left(\theta^{\frac{\ell+1}{2}} G(z)\right)=\omega(\theta G(z)) .
$$

Thus, from (3.2) we have

$$
\frac{\ell+1}{2} \geq \alpha
$$

Note that $\omega\left(\theta^{\ell-r+2} G(z)\right) \equiv 1+\alpha(\bmod \ell)$ and $1+\alpha>0$. Since

$$
0<1+\alpha+r-\frac{\ell+3}{2} \leq \ell,
$$

Lemma 2.1 (6) implies that

$$
\omega(\theta G(z))=\omega\left(\theta^{\frac{\ell+1}{2}} G(z)\right)=\omega\left(\theta^{\ell-r+2} G(z)\right)+\left(r-\frac{\ell+3}{2}\right)(\ell+1) .
$$

Thus, we have

$$
\alpha=\frac{\ell+1}{2} .
$$

Noting that $\omega\left(\theta^{\ell-r+2} G(z)\right)>0$ by Lemma 2.1 (1), we have

$$
\omega(\theta G(z))>(\ell+1)\left(r-\frac{\ell+3}{2}\right) .
$$

Since $\omega(\theta G(z)) \leq 2 \ell$, we have

$$
r \leq \frac{\ell+5}{2}
$$

Combining (3.1) and Lemma 2.1 (6), we have $\omega(\theta G(z))=\ell+r=\omega(G(z))+\ell+1$. This completes the proof. $\ell$.

In the following lemma, we prove that $f(z) \mid U_{\ell^{m}}$ is not equal to a constant modulo 
Lemma 3.2. Suppose that $\lambda$ is an integer, that $N$ is a positive integer $4 \mid N$, and $\ell \geq 5$ is a prime such that $\ell \nmid N$. Let

$$
f(z):=\sum a(n) q^{n} \in \mathcal{M}_{\lambda+\frac{1}{2}}\left(\Gamma_{0}(N)\right) \cap\left(\mathbb{Z}\left[q^{-1}\right]\right)[[q]] .
$$

If $a(0) \not \equiv 0(\bmod \ell)$, then

$$
f(z) \mid U_{\ell} \not \equiv a(0) \quad(\bmod \ell) .
$$

Proof of Lemma 3.2. Suppose that $f(z) \mid U_{\ell} \equiv a(0)(\bmod \ell)$. Take a cusp form $g(z)$ $\in S_{\lambda+\frac{1}{2}}\left(\Gamma_{0}(N)\right) \cap \mathbb{Z}[[q]]$ such that $f(z) g(z)$ is holomorphic at all the cusps of $\Gamma_{0}(N)$. Since

$$
f(z) g(z)^{\ell}\left|U_{\ell} \equiv f(z)\right| U_{\ell} \cdot g(z) \equiv a(0) g(z) \quad(\bmod \ell),
$$

we have

$$
a(0)^{2} g(z)^{2} \equiv f(z)^{2}\left(g(z)^{\ell} \mid U_{\ell}\right)^{2} \quad(\bmod \ell) .
$$

Thus, the difference of the weights of $g(z)^{2}$ and $f(z)^{2}\left(g(z)^{\ell} \mid U_{\ell}\right)^{2}$ is divisible by $\ell-1$. This contradicts that the weights of $g(z)^{2}$ and $f(z)^{2}\left(g(z)^{\ell} \mid U_{\ell}\right)^{2}$ are odd and even, respectively. Thus, we complete the proof.

Using the following lemma, we can take a positive integer $\alpha$ such that

$$
\omega\left(f(z) \mid U_{\ell^{\alpha}} \cdot T(z)^{\ell}\right) \leq \ell+\frac{\ell+1}{2} .
$$

Lemma 3.3. Suppose that $\lambda$ is a nonnegative integer, that $N$ is a positive integer $4 \mid N$, and $\ell \geq 5$ is a prime such that $\ell \nmid N$. Suppose that

$$
f(z):=\sum_{n=0}^{\infty} a(n) q^{n} \in M_{\lambda+\frac{1}{2}}\left(\Gamma_{0}(N)\right) \cap \mathbb{Z}[[q]] .
$$

If $\alpha \geq 2\left[\frac{\lambda}{\ell-1}\right]$, then

$$
\ell+\frac{\ell+1}{2} \geq \omega\left(f(z) \mid U_{\ell^{\alpha}} \cdot T(z)^{\ell}\right) .
$$

Proof of Lemma 3.3. Note that

$$
\theta\left(f(z) T(z)^{\ell}\right) \equiv \theta f(z) \cdot T(z)^{\ell} \quad(\bmod \ell)
$$

and

$$
\omega(\theta(f(z))) \leq \omega(f(z))+\ell+1 .
$$

Thus, using Lemma 2.1 (4) and (6), we have

$$
\omega\left(f(z) T(z)^{\ell}-\theta^{\ell-1} f(z) \cdot T(z)^{\ell}\right) \leq(\ell+1)(\ell-1)+\omega\left(f(z) T(z)^{\ell}\right) .
$$

Since

$$
\left(f(z) \mid U_{\ell} \cdot T(z)\right)^{\ell} \equiv f(z) T(z)^{\ell}-\theta^{\ell-1} f(z) T(z)^{\ell} \quad(\bmod \ell)
$$

and $\left(f(z) T(z)^{\ell}\right)\left|U_{\ell} \equiv f(z)\right| U_{\ell} \cdot T(z)(\bmod \ell)$, we have

$$
\begin{aligned}
\omega\left(f(z) \mid U_{\ell} \cdot T(z)^{\ell}\right) & =\omega\left(f(z) \mid U_{\ell} \cdot T(z)\right)+\omega\left(T(z)^{\ell-1}\right) \\
& \leq(\ell+1) \frac{\ell-1}{\ell}+\frac{1}{\ell} \cdot \omega\left(f(z) \cdot T(z)^{\ell}\right)+\frac{\ell-1}{2} .
\end{aligned}
$$

Thus, we have

$$
\begin{array}{ll}
\omega\left(f(z) \mid U_{\ell} \cdot T(z)^{\ell}\right)<\omega\left(f(z) \cdot T(z)^{\ell}\right) & \text { if } \omega\left(f(z) T(z)^{\ell}\right)>\ell+\frac{\ell+1}{2} \\
\omega\left(f(z) \mid U_{\ell} \cdot T(z)^{\ell}\right) \leq \ell+\frac{\ell+1}{2} & \text { if } \omega\left(f(z) \cdot T(z)^{\ell}\right) \leq \ell+\frac{\ell+1}{2} .
\end{array}
$$


Note that $\omega\left(f(z) T(z)^{\ell}\right)-\omega\left(f(z) \mid U_{\ell} \cdot T(z)^{\ell}\right) \equiv 0\left(\bmod \frac{\ell-1}{2}\right)$. This completes the proof.

With the previous lemmas, we can prove Theorem 2, Our argument for the proof of Theorem 2 is based on the work of Bruinier and Ono [7] and of Ahlgren and Boylan [1], [3], 4].

Proof of Theorem 2. First, we assume that $\operatorname{gcd}(\ell, N)=1$. Let

$$
G_{\ell}(z):=\frac{\eta(4 z)^{\ell^{2}}}{\eta\left(4 \ell^{2} z\right)} \in M_{\frac{\ell^{2}-1}{2}}\left(\Gamma_{0}\left(4 \ell^{2}\right)\right) .
$$

By the well-known properties of eta-quotients (see [10]) and Proposition 3.2 in [18], there exist positive integers $r_{0}, v_{0}$ such that for every integer $r \geq r_{0}$ and $v \geq v_{0}$

$$
\begin{gathered}
\left(f(z) \mid U_{\ell^{r}}\right) G_{\ell}(z)^{\ell^{v}} \in M_{\lambda^{\prime}+\frac{1}{2}}\left(\Gamma_{0}\left(\ell^{2} N\right)\right), \\
\left(f(z)\left|U_{\ell^{r}}-f(z)\right| U_{\ell^{r+1}} \mid V_{\ell}\right) G_{\ell}(z)^{\ell^{v}} \in S_{\lambda^{\prime}+\frac{1}{2}}\left(\Gamma_{0}\left(\ell^{2} N\right)\right) .
\end{gathered}
$$

Note that

$$
\begin{aligned}
\left(f(z) \mid U_{\ell^{r}}\right) G_{\ell}(z)^{\ell^{v}} & \equiv f(z) \mid U_{\ell^{r}} \quad\left(\bmod \ell^{j}\right), \\
\left(f(z)\left|U_{\ell^{r}}-f(z)\right| U_{\ell^{r+1}} \mid V_{\ell}\right) G_{\ell}(z)^{\ell^{v}} & \equiv f(z)\left|U_{\ell^{r}}-f(z)\right| U_{\ell^{r+1}} \mid V_{\ell} \quad\left(\bmod \ell^{j}\right),
\end{aligned}
$$

and that

$$
\lambda^{\prime} \equiv \lambda \quad(\bmod \ell-1)
$$

Following the argument of Theorem 3.37 of [13, we have that, for each integer $r \geq r_{0}$, there are finitely many square-free integers $n_{1}, n_{2}, \ldots, n_{t}$ such that

$$
\theta\left(f(z) \mid U_{\ell^{r}}\right) \equiv \sum_{i=1}^{t} \sum_{m=1}^{\infty} a\left(n_{i} m^{2} \ell^{r}\right) n_{i} m^{2} q^{n_{i} m^{2}} \quad(\bmod \ell) .
$$

From Proposition 5.1 of 4 ] there exists a modular form $F(z) \in M_{k+\frac{1}{2}}\left(\Gamma_{0}(N)\right) \cap \mathbb{Z}[[q]]$ such that

$$
F(z) \equiv f(z) \mid U_{\ell^{r}} \quad(\bmod \ell)
$$

where $k+\frac{1}{2}=\left(\lambda^{\prime}+\frac{1}{2}\right) \ell^{2 m}+\ell^{e}(\ell-1)$ for sufficiently large $e, m \in \mathbb{N}$. Note that

$$
k \equiv \lambda^{\prime} \quad(\bmod \ell-1)
$$

Using Lemma 3.3. there exists a positive integer $\alpha$ such that

$$
\omega\left(F(z) \mid U_{\ell^{\alpha}} \cdot T(z)^{\ell}\right)<\ell+\frac{\ell+1}{2} .
$$

Moreover, Lemma 3.2 implies that

$$
F(z) \mid U_{\ell^{\alpha}} \not \equiv a(0) \quad(\bmod \ell) .
$$

If $\theta\left(F(z) \mid U_{\ell^{\alpha}} \cdot T(z)^{\ell}\right) \equiv 0(\bmod \ell)$, then $\omega\left(F(z) \mid U_{\ell^{\alpha}} \cdot T(z)^{\ell}\right) \equiv 0(\bmod \ell)$. Thus, from (3.5) we have $\omega\left(F(z) \mid U_{\ell^{\alpha}} \cdot T(z)^{\ell}\right)=\ell$. This implies that $\lambda \equiv 0\left(\bmod \frac{\ell-1}{2}\right)$.

From now on we assume that

$$
\theta\left(F(z) \mid U_{\ell^{\alpha}}\right) \not \equiv 0 \quad(\bmod \ell) .
$$

Let

$$
\omega\left(F(z) \mid U_{\ell^{\alpha}} \cdot T(z)^{\ell}\right)=\bar{\lambda}+\frac{\ell+1}{2} .
$$


From (3.4) there are finitely many square-free integers $n_{1}, n_{2}, \ldots, n_{t}$ such that

$$
\theta\left(F(z) \mid U_{\ell^{\alpha}}\right) \equiv \sum_{i=1}^{t} \sum_{m=1}^{\infty} a\left(n_{i} m^{2} \ell^{\alpha}\right) n_{i} m^{2} q^{n_{i} m^{2}} \quad(\bmod \ell) .
$$

Let $\chi$ be a real Dirichlet character modulo a prime $p \nmid N \ell$. Note that

$$
\begin{gathered}
F(z) \otimes \chi \in M_{k+\frac{1}{2}}\left(\Gamma_{0}\left(N p^{2}\right)\right) \cap \mathbb{Z}[[q]], \\
F(z) \mid U_{p} \in M_{k+\frac{1}{2}}\left(\Gamma_{0}\left(N p,\left(\frac{4 p}{\bullet}\right)\right)\right) \cap \mathbb{Z}[[q]]
\end{gathered}
$$

(see Chapter 3 in [13]). Here, $\left(\frac{4 p}{\bullet}\right)$ denotes the Jacobi symbol. Thus, by Lemma 3.3 we have

$$
\begin{aligned}
\max \left\{\omega\left(F(z) \mid U_{\ell^{\alpha}} \cdot T(z)^{\ell}\right), \omega\right. & \left(\left(F(z) \mid U_{p}\right) \mid U_{\ell^{\alpha}} \cdot T(z)^{\ell}\right), \\
& \left.\omega\left((F(z) \otimes \chi) \mid U_{\ell^{\alpha}} \cdot T(z)^{\ell}\right)\right\} \leq \ell+\frac{\ell+1}{2} .
\end{aligned}
$$

Let

$$
\begin{aligned}
\bar{\lambda}:=\max & \left\{\omega\left(F(z) \mid U_{\ell^{\alpha}} \cdot T(z)^{\ell}\right),\right. \\
& \left.\omega\left(\left(F(z) \mid U_{p}\right) \mid U_{\ell^{\alpha}} \cdot T(z)^{\ell}\right), \omega\left((F(z) \otimes \chi) \mid U_{\ell^{\alpha}} \cdot T(z)^{\ell}\right)\right\} .
\end{aligned}
$$

Following the argument of Lemma 4.1 of [4], we can find an integer $n_{v} \in\left\{n_{1}, \ldots, n_{t}\right\}$, primes $p_{v, 1}, \ldots, p_{v, s_{v}}$ greater than $\ell$, and a modular form $F_{v}(z)$ with the following properties:

(1) $F_{v}(z) \in M_{\bar{\lambda}}\left(\Gamma_{0}\left(N n_{v}^{\prime} p_{v, 1}^{2} \cdots p_{v, s_{v}}^{2},\left(\frac{4 n_{v}^{\prime}}{\bullet}\right) \chi\right)\right) \cap \mathbb{Z}[[q]]$, $\operatorname{gcd}\left(N n_{v}^{\prime} p_{v, 1}^{2} \cdots p_{v, s_{v}}^{2}, \ell\right)=1$, where $n_{v}:=n_{v}^{\prime} \ell^{u}$ and $\operatorname{gcd}\left(\ell, n_{v}^{\prime}\right)=1$,

(2) $\theta F_{v}(z) \equiv\left(\sum_{\operatorname{gcd}\left(m, \prod_{j} p_{v, j}\right)=1} a\left(n_{v} \ell^{\alpha} m^{2}\right) m^{2} q^{m^{2}}\right) \cdot T(z)^{\ell}(\bmod \ell)$,

(3) $\theta F_{v}(z) \not \equiv 0(\bmod \ell)$.

Note that

$$
\ell \nmid N n_{v}^{\prime} p_{v, 1}^{2} \cdots p_{v, s_{v}}^{2},
$$

and that $\bar{\lambda} \equiv k+\frac{\ell+1}{2} \equiv \lambda+\frac{\ell+1}{2}\left(\bmod \frac{\ell-1}{2}\right)$. Lemma 3.1 implies that

$$
\lambda \equiv 0 \text { or } 1 \quad\left(\bmod \frac{\ell-1}{2}\right) .
$$

This completes the proof of Theorem 2 (1).

Now we treat the case when $j=1$ and $\ell \mid N$. Let $N:=N^{\prime} \ell^{m}$, where $\operatorname{gcd}\left(N^{\prime}, \ell\right)=$ 1. Note that $\eta(z)^{24} \in S_{12}(\Gamma(1))$. We can take a positive integer $b$ such that $\eta(z)^{24 b} f(z)$ is a holomorphic modular form of weight $\lambda+12 b+\frac{1}{2}$. Using Proposition 5.1 in [4], we have that there exists a modular form $G(z) \in M_{k+\frac{1}{2}}\left(\Gamma_{0}\left(N^{\prime}\right)\right) \cap \mathbb{Z}[[q]]$ such that

$$
G(z) \equiv \eta(z)^{24 b} f(z) \quad(\bmod \ell)
$$

where $k+\frac{1}{2}=\left(\lambda+12 b+\frac{1}{2}\right) \ell^{2 m}+\ell^{e}(\ell-1)$ for sufficiently large $e, m \in \mathbb{N}$. Note that

$$
k \equiv \lambda+12 b \quad(\bmod \ell-1) .
$$

Since $\eta(z)^{24 b}$ is holomorphic on the complex upper half-plane, the function $\frac{G(z)}{\eta(z)^{24 b}}$ is a weakly holomorphic modular form. Note that $\frac{G(z)}{\eta(z)^{24 b}} \equiv f(z)(\bmod \ell)$ and that the weight of $\frac{G(z)}{\eta(z)^{24 b}}$ is equal to that of $f(z)$ modulo $\ell-1$. This completes the proof by Theorem 2 (1). 


\section{Proofs of Theorems 1 and 3}

First, we prove Theorem 1 .

Proof of Theorem 1. Let $N:=N^{\prime} \ell^{e}$, where $\operatorname{gcd}\left(N^{\prime}, \ell\right)=1$. From the assumption, let

$$
f(z) \equiv a(0)+\sum_{i=1}^{t} \sum_{m=1}^{\infty} a\left(n_{i} m^{2}\right) q^{n_{i} m^{2}} \quad(\bmod \ell) .
$$

Following the argument of the proof of Theorem 2, we can find an integer $n_{v} \in$ $\left\{n_{1}, \ldots, n_{t}\right\}$, primes $p_{v, 1}, \ldots, p_{v, s_{v}}$ greater than $\ell$, and a modular form $F_{v}(z)$ with the following properties:

(1) $F_{v}(z) \in M_{\bar{\lambda}}\left(\Gamma_{0}\left(N^{\prime} n_{v}^{\prime} p_{v, 1}^{2} \cdots p_{v, s_{v}}^{2},\left(\frac{4 n_{v}^{\prime}}{\bullet}\right) \chi\right)\right) \cap \mathbb{Z}[[q]]$, $\operatorname{gcd}\left(N n_{v}^{\prime} p_{v, 1}^{2} \cdots p_{v, s_{v}}^{2}, \ell\right)=1$, where $n_{v}:=n_{v}^{\prime} \ell^{u}$ and $\operatorname{gcd}\left(\ell, n_{v}^{\prime}\right)=1$,

(2) $\theta F_{v}(z) \equiv\left(\sum_{\operatorname{gcd}\left(m, \prod_{j} p_{v, j}\right)=1} a\left(n_{v} m^{2} \ell^{\alpha}\right) m^{2} q^{m^{2}}\right) \cdot T(z)^{\ell}(\bmod \ell)$, where $\alpha$ is a nonnegative integer,

(3) $\theta F_{v}(z) \not \equiv 0(\bmod \ell)$,

(4) $\bar{\lambda} \leq \ell+\frac{\ell+1}{2}, \bar{\lambda} \equiv \lambda+\frac{\ell+1}{2}\left(\bmod \frac{\ell+1}{2}\right)$.

This completes the proof by Lemma 3.1 .

Following the arguments of Kiming and Olsson [12, we prove Theorem 3 ,

Proof of Theorem 13. Let $N:=N^{\prime} \ell^{m}$, where $\operatorname{gcd}\left(N^{\prime}, \ell\right)=1$. Suppose that there is a congruence for $f(z)$ at $(\ell, \beta), 0 \leq \beta<\ell$. Following the argument of Theorem 2 (2), we have that there exists a modular form $g(z) \in M_{\lambda^{\prime}+\frac{1}{2}}\left(\Gamma_{0}\left(N^{\prime}\right)\right)$ satisfying the following:

(1) $g(z) \equiv f(z) \mid U_{\ell^{\alpha}}(\bmod \ell)$ for some positive integer $\alpha$,

(2) $\lambda^{\prime} \equiv \lambda(\bmod \ell-1)$,

(3) $g(z) \not \equiv a(0)(\bmod \ell)$

(4) $\omega\left(g(z) T^{\ell}(z)\right) \leq \ell+\frac{\ell+1}{2}$.

Note that there is a congruence for $g(z)$ at $(\ell, \beta)$, and that $\beta \neq 0$ by Lemma 3.2 . This implies that $\left(q^{-\beta} g(z) T(z)^{\ell}\right) \mid U_{\ell} \equiv 0(\bmod \ell)$. Thus, we have

$$
\left(q^{-\beta} g(z) T(z)^{\ell}\right) \equiv \theta^{\ell-1}\left(q^{-\beta} g(z) T(z)^{\ell}\right) \quad(\bmod \ell) .
$$

Since $\theta^{\ell-1}\left(q^{-\beta} g(z) T(z)^{\ell}\right) \equiv q^{-\beta} \sum_{i=0}^{\ell-1} \beta^{\ell-1-i} \theta^{i}\left(g(z) T(z)^{\ell}\right)$ ( $\left.\bmod \ell\right)$, we have

$$
\sum_{i=1}^{\ell-1} \beta^{\ell-1-i} \theta^{i}\left(g(z) T(z)^{\ell}\right) \equiv 0 \quad(\bmod \ell) .
$$

Note that

$$
\omega\left(\theta^{i}\left(g(z) T(z)^{\ell}\right)\right) \equiv \omega\left(\theta^{i+\frac{\ell-1}{2}}\left(g(z) T(z)^{\ell}\right)\right) \equiv \omega\left(g(z) T(z)^{\ell}\right)+2 i \quad(\bmod \ell-1)
$$

for $i=0,1, \ldots, \frac{\ell-1}{2}$. Thus for every $i \in\left\{0,1, \ldots, \frac{\ell-1}{2}\right\}$ we have

$$
\beta^{\ell-1-i} \theta^{i}\left(g(z) T(z)^{\ell}\right)+\beta^{\frac{\ell-1}{2}-i} \theta^{i+\frac{\ell-1}{2}}\left(g(z) T(z)^{\ell}\right) \equiv 0 \quad(\bmod \ell) .
$$


This implies that $\beta^{\ell-1-i} \theta^{i}\left(g(z) T(z)^{\ell}\right) \equiv-\beta^{\frac{\ell-1}{2}-i} \theta^{i+\frac{\ell-1}{2}}\left(g(z) T(z)^{\ell}\right)(\bmod \ell)$. Since $\beta \neq 0$, we have $\omega\left(\theta^{i}\left(g(z) T(z)^{\ell}\right)\right)=\omega\left(\theta^{i+\frac{\ell-1}{2}}\left(g(z) T(z)^{\ell}\right)\right)$. This completes the proof by Lemma 3.1 .

\section{ACKNOWLEDGEMENT}

The author thanks Professor S. Ahlgren for helpful comments and also the referee for useful comments. This work was supported by Korea Research Foundation Grant funded by the Korean Government (KRF-2008-331-C00005).

\section{REFERENCES}

[1] S. Ahlgren and M. Boylan, Arithmetic properties of the partition function, Invent. Math. 153 (2003), no. 3, 487-502. MR2000466 (2004e:11115)

[2] S. Ahlgren and M. Boylan, Coefficients of half-integral weight modular forms modulo $l^{j}$, Math. Ann. 331 (2005), no. 1, 219-239. MR2107445 (2005k:11091)

[3] Scott Ahlgren and Matthew Boylan, Addendum: "Coefficients of half-integral weight modular forms modulo $l^{j}$ " [Math. Ann. 331 (2005), no. 1, 219-239], Math. Ann. 331 (2005), no. 1, 241-242.

[4] S. Ahlgren and M. Boylan, Central Critical Values of Modular L-functions and Coeffients of Half Integral Weight Modular Forms Modulo $\ell$, Central critical values of modular $L$-functions and coefficients of half-integral weight modular forms modulo $l$. Amer. J. Math. 129 (2007), no. 2, 429-454. MR:2306041(2008c:11075)

[5] Antal Balog, Henri Darmon and Ken Ono, Congruence for Fourier coefficients of half-integral weight modular forms and special values of L-functions (English summary). Analytic number theory, Vol. 1 (Allerton Park, IL, 1995), 105-128, Progr. Math., 138, Birkhauser Boston, Boston, MA, 1996. MR.1399333 (97e:11056)

[6] Jan Hendrik Bruinier, Non-vanishing modulo $\ell$ of Fourier coefficients of half-integral weight modular forms, Duke Math. J. 98 (1999), no. 3, 595-611. MR1695803 (2000d:11061)

[7] J. H. Bruinier and K. Ono, Coefficients of half-integral weight modular forms, J. Number Theory 99 (2003), no. 1, 164-179. MR.1957250 (2004b:11056)

[8] D. Choi and T. Kilbourn, The weight of half-integral weight modular forms with few nonvanishing coefficients mod $\ell$, Acta Arith. 127 (2007), 193-197. MR2289984 (2007m:11066)

[9] S. Corteel and J. Lovejoy, Overpartitions, Trans. Amer. Math. Soc. 356 (2004) 1623-1635. MR.2034322 (2004j:11118)

[10] B. Gordon and K. Hughes, Multiplicative properties of eta-products. II, Cont. Math. 143 (1993), 415-430. MR1210529 (94a:11058)

[11] Benedict H. Gross, A tameness criterion for Galois representations attached to modular forms $(\bmod p)$, Duke Math. J. 61 (1990), no. 2, 445-517. MR1074305 (91i:11060)

[12] I. Kiming and J. Olsson, Congruences like Ramanujan's for powers of the partition function, Arch. Math. (Basel) 59 (1992), 348-360. MR1179461 (93g:11105)

[13] Ken Ono, The web of modularity: arithmetic of the coefficients of modular forms and q-series. volume 102 of CBMS Regional Conference Series in Mathematics, Published for the Conference Board of the Mathematical Sciences, Washington, DC, 2004. MR2020489|(2005c:11053)

[14] Ken Ono, Christopher Skinner, Fourier coefficients of half-integral weight modular forms modulo l, Ann. of Math. (2) 147 (1998), no. 2, 453-470. 1998. MR1626761 (99f:11059a)

[15] Ken Ono, Christopher Skinner, Corrigendum: "Fourier coefficients of half-integral weight modular forms modulo l", Ann. of Math. (2) 148 (1998), no. 1, 361. MR.1652912 (99f:11059b)

[16] Jean-Pierre Serre, Formes modulaires et fonctions zeta p-adiques (French). Modular functions of one variable, III (Proc. Internat. Summer School, Univ. Antwerp, 1972), pp. 191-268. Lecture Notes in Math., Vol. 350, Springer, Berlin, 1973. MR0404145 (53:7949a)

[17] H. P. F. Swinnerton-Dyer, On l-adic representations and congruences for coefficients of modular forms. Modular functions of one variable, III (Proc. Internat. Summer School, Univ. Antwerp, 1972), pp. 1-55. Lecture Notes in Math., Vol. 350, Springer, Berlin, 1973. MR0406931(53:10717a) 
[18] S. Treneer, Congruences for the Coefficients of Weakly Holomorphic Modular Forms, Proc. London Math. Soc., (3) 93 (2006), 304-324. MR2251155 (2007c:11058)

[19] M. F. Vigneras, Facteurs gamma et equations fonctionelles, Lecture Notes in Math. vol. 627 (Modular functions of one variable VI) (1977), Springer-Verlag, Berlin, pp. 79-103. MR0485739(58:5552)

School of Liberal Arts and Sciences, Korea Aerospace University, 200-1, HwajeonDong, Goyang, Gyeonggi, 412-791, Korea

E-mail address: choija@postech.ac.kr 